\title{
Low p-type contact resistance by field-emission tunneling in highly Mg-doped GaN
}

Hironori Okumura, Denis Martin, and Nicolas Grandjean

Citation: Appl. Phys. Lett. 109, 252101 (2016); doi: 10.1063/1.4972408

View online: http://dx.doi.org/10.1063/1.4972408

View Table of Contents: http://aip.scitation.org/toc/apl/109/25

Published by the American Institute of Physics

\section{Articles you may be interested in}

Undoped p-type GaN1-xSbx alloys: Effects of annealing

Appl. Phys. Lett. 109, 252102252102 (2016); 10.1063/1.4972559

Deep ultraviolet emission from ultra-thin GaN/AIN heterostructures

Appl. Phys. Lett. 109, 241102241102 (2016); 10.1063/1.4971968

The effect of polarity on MOCVD growth of thick InGaN

Appl. Phys. Lett. 110, 022101022101 (2017); 10.1063/1.4972967

Ultra-fine metal gate operated graphene optical intensity modulator

Appl. Phys. Lett. 109, 251101251101 (2016); 10.1063/1.4972306

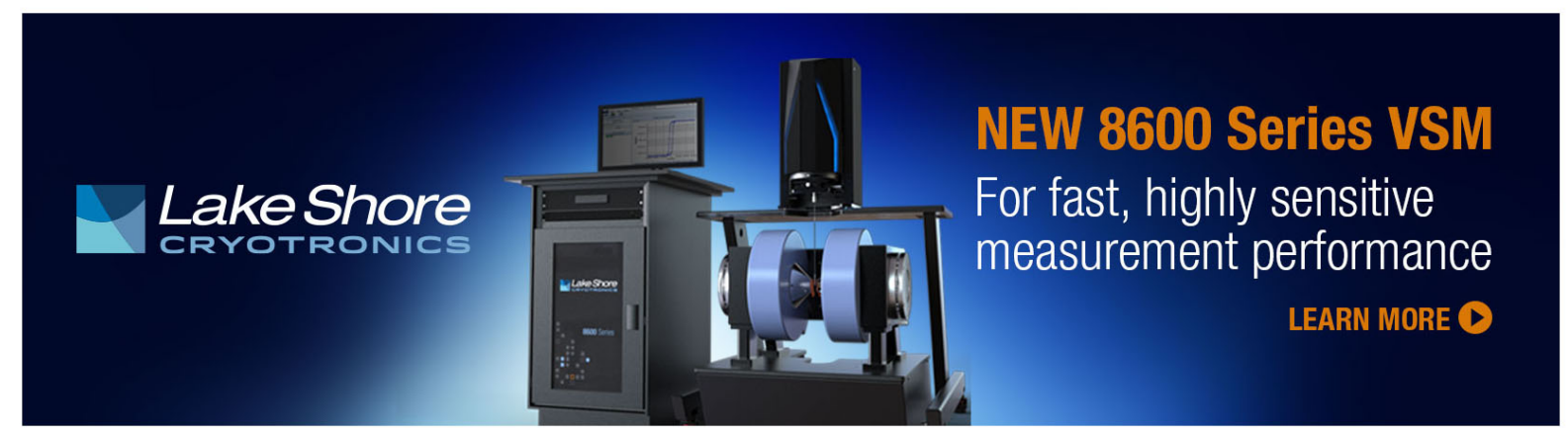




\title{
Low $p$-type contact resistance by field-emission tunneling in highly Mg-doped GaN
}

\author{
Hironori Okumura, ${ }^{1,2, a)}$ Denis Martin, ${ }^{2}$ and Nicolas Grandjean ${ }^{2}$ \\ ${ }^{1}$ Institute of Applied Physics, University of Tsukuba, 305-8573 Ibaraki, Japan \\ ${ }^{2}$ Institute of Physics, École Polytechnique Fédérale de Lausanne (EPFL), CH-1015 Lausanne, Switzerland
}

(Received 15 August 2016; accepted 5 December 2016; published online 19 December 2016)

\begin{abstract}
Mg-doped GaN with a net acceptor concentration $\left(N_{A^{-}} N_{D}\right)$ in the high $10^{19} \mathrm{~cm}^{-3}$ range was grown using ammonia molecular-beam epitaxy. Electrical properties of $\mathrm{NiO}$ contact on this heavily doped $p$-type $\mathrm{GaN}$ were investigated. A potential-barrier height of $0.24 \mathrm{eV}$ was extracted from the relationship between $N_{A}-N_{D}$ and the specific contact resistivity $\left(\rho_{c}\right)$. We found that there is an optimum $N_{A}-N_{D}$ value of $5 \times 10^{19} \mathrm{~cm}^{-3}$ for which $\rho_{c}$ is as low as $2 \times 10^{-5} \Omega \mathrm{cm}^{2}$. This low $\rho_{c}$ is ascribed to hole tunneling through the potential barrier at the $\mathrm{NiO} / p^{+}-\mathrm{GaN}$ interface, which is well accounted for by the field-emission model. Published by AIP Publishing.

[http://dx.doi.org/10.1063/1.4972408]
\end{abstract}

High-voltage power and low-loss switching devices are attractive for high-voltage power supplies, single-phase converters, and solid-state transformers. The switching performance of high-power devices is evaluated by the materials-based figure of merits (FOMs), which are associated with the blocking voltage and the on-resistance. GaN devices exhibit superior FOM in comparison with $\mathrm{SiC}$ counterparts primarily due to high-breakdown electric fields $(\sim 3 \mathrm{MV} / \mathrm{cm})$, leading to great potential for high-power applications. Recently, GaN-power devices based on vertical structures, such as PIN rectifiers, have received much attention owing to the improvement of GaN epitaxial growth. Vertical devices enable both high blocking voltages by tailoring the electric-field distribution through a drift region and low on-resistance by the high injection of minority carriers into the drift region, i.e., conduction modulation. GaN PIN diodes with blocking voltages above $4 \mathrm{kV}$ have been reported. ${ }^{1,2}$ However, the forward-bias voltage of these GaN PIN diodes had a differential on-resistance of $2-3 \mathrm{~m} \Omega \mathrm{cm}^{2}$, which is comparable to $\mathrm{SiC}$ PIN diodes with a much higher blocking-voltage of $13 \mathrm{kV}$ despite the trade-off relation between the blocking voltage and the drift resistance. ${ }^{3}$ The on-resistance of vertical devices is mainly composed of contact, drift, and substrate resistances. ${ }^{4}$ Still GaN PIN diodes suffer from high $p$-type contact resistance. Although very low $p$-type contact resistivity, in the $10^{-6} \Omega \mathrm{cm}^{2}$ range, has been reported, ${ }^{5-7}$ still in GaN devices they are in the $10^{-4} \Omega \mathrm{cm}^{2}$ range. ${ }^{8,9}$ Thus, a further reduction of $p$-type contact resistance would significantly improve the performance of GaN PIN diodes in the view of energy savings.

The voltage drop depends on the height of the potential barrier $\left(\Phi_{B}\right)$, which is formed at the metal-semiconductor interface due to the difference between the metal work function and the semiconductor electron affinity. Low $\Phi_{B}$ is required to achieve ohmic behavior. Pd and oxidized Ni contact layers on $p$-type $\mathrm{GaN}$ exhibit fairly low $\Phi_{B}$, typically $0.2-0.5 \mathrm{eV} .^{10-12}$ Thus, further reducing the contact resistance requires that the top-most surface of the semiconductor is

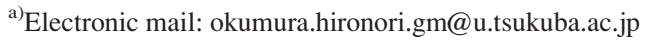

heavily doped in order to decrease the depletion-region width and to allow tunneling through the potential barrier. Usually, $p$-type GaN contact layers feature a high $\mathrm{Mg}$ concentration $[\mathrm{Mg}]$ of $\sim 10^{20} \mathrm{~cm}^{-3} \cdot{ }^{13}$ However, achieving a net acceptor concentration $\left(N_{A}-N_{D}\right)$ and hole concentration in excess of $10^{19} \mathrm{~cm}^{-3}$ is quite challenging because of the high ionization energy of $\mathrm{Mg}(\sim 180 \mathrm{meV})$ and $\mathrm{Mg}$-acceptor compensation by donor-like defects, causing high contact and sheet resistances. ${ }^{14}$ Low growth temperature and nitrogenrich conditions are preferable to minimize the compensation defects in $p$-type GaN. Molecular-beam epitaxy (MBE) has an advantage with low growth temperature. GaN growth using plasma-assisted MBE has a tendency to produce rough surfaces and a high defect density for low growth temperatures $\left(<750^{\circ} \mathrm{C}\right)$ under nitrogen-rich conditions due to small adatom diffusion. ${ }^{15,16}$ By enhancing adatom diffusion using metal modulation epitaxy (MME), Trybus et al. grew $\mathrm{GaN}$ with high crystalline quality at a low growth temperature of $500^{\circ} \mathrm{C}$ under nitrogen-rich conditions, achieving a high hole concentration of $2 \times 10^{19} \mathrm{~cm}^{-3} \cdot{ }^{17}$ In contradiction to plasma-assisted MBE, GaN growth using ammonia $\left(\mathrm{NH}_{3}\right)$ MBE has high crystalline quality under nitrogen-rich conditions without MME. Recently, we achieved p-type $\mathrm{GaN}$ layers with low compensation defects, i.e., less than $3 \times 10^{17} \mathrm{~cm}^{-3}$ and high $N_{A}-N_{D}\left(7 \times 10^{19} \mathrm{~cm}^{-3}\right)$ by growing GaN:Mg at low temperature using ammonia MBE. ${ }^{18,19}$ Such high doping levels should enable very low $p$-type contact resistance.

In this paper, we report on a study of the contact resistance of $\mathrm{NiO}$ on heavily doped $p$-type $\mathrm{GaN}$ grown by $\mathrm{NH}_{3}$ MBE. We show that low contact resistance can be achieved, which is attributed to hole tunneling through the potential barrier at the $\mathrm{NiO} / p^{+}-\mathrm{GaN}$ interface.

$\mathrm{GaN}$ epilayers were deposited by $\mathrm{NH}_{3}-\mathrm{MBE}$ on $4-\mu \mathrm{m}-$ thick $\mathrm{GaN}$ (0001) templates grown on c-plane sapphire substrates by metal-organic vapor phase epitaxy (MOVPE). After a $0.5-\mu$ m-thick unintentionally doped GaN buffer, a $0.5 \mu$ m-thick $\mathrm{Mg}$-doped $\mathrm{GaN}$ layer was deposited at $740^{\circ} \mathrm{C}$ using an MBE system equipped with purified ammonia gas. The Mg doping level was controlled by a valved cracker 
cell with a beam equivalent pressure varying between $0.9 \times 10^{-10}$ and $4.2 \times 10^{-10}$ Torr, corresponding to $[\mathrm{Mg}]$ between $8 \times 10^{18}$ and $2 \times 10^{20} \mathrm{~cm}^{-3}$. Detailed growth conditions are reported in Ref. 19. The root-mean-square surface roughness determined by atomic force microscopy on a $5 \times 5 \mu \mathrm{m}^{2}$ scan was $5 \pm 1 \mathrm{~nm}$ for all GaN:Mg layers. After the growth, GaN:Mg layers were ultrasonically cleaned with acetone and isopropyl alcohol, rinsed with deionized water, and dipped in $\mathrm{KOH}$ at $120^{\circ} \mathrm{C}$ for $1 \mathrm{~min}$ to remove residual oxidation on the surface. ${ }^{20}$ This cleaning step was followed by a deposition of $\mathrm{Ni}(25 \mathrm{~nm}) / \mathrm{Au}(25 \mathrm{~nm})$ metal stack by electron-beam evaporation. $\mathrm{Ni} / \mathrm{Au}$ contacts were then annealed at $500{ }^{\circ} \mathrm{C}$ for $10 \mathrm{~min}$ in an oxygen atmosphere to form $\mathrm{NiO}^{21} \mathrm{We}$ grew $\mathrm{GaN}: \mathrm{Mg}$ layers with various [Mg]. $N_{A}-N_{D}$ was measured by electrochemical capacitancevoltage measurements. Sheet resistance $\left(R_{s}\right)$ and specific contact resistivity $\left(\rho_{c}\right)$ of $\mathrm{GaN}: \mathrm{Mg}$ layers were extracted using transfer length measurements (TLMs) at room temperature. For each GaN:Mg layer, we prepared two samples with the same device processing to improve the data reliability. We measured $R_{s}$ and $\rho_{c}$ at five different positions on both samples. Hole concentration and mobility were determined by Hall-effect measurements.

We previously found that $N_{A}-N_{D}$ almost corresponds to $[\mathrm{Mg}]$ up to a critical concentration of $7 \times 10^{19} \mathrm{~cm}^{-3}$ and then dramatically reduces for higher $[\mathrm{Mg}] .{ }^{19}$ Similar behavior has been observed on MOVPE grown GaN:Mg layers, but with a lower critical acceptor concentration of $3 \times 10^{19} \mathrm{~cm}^{-3} \cdot 22$ The higher net acceptor concentration measured on MBE grown layers is ascribed to a lower growth temperature, which either hinders the introduction of compensation centers or avoids $\mathrm{Mg}$ species to turn into compensating interstitial site states. ${ }^{23}$ Current-voltage $(I-V)$ characteristics of $p$-type GaN layers with $\mathrm{NiO}$ contacts are displayed in Fig. 1(a) for various $[\mathrm{Mg}]$. We can tentatively classify the electrical characteristics into three categories depending on $[\mathrm{Mg}]$.
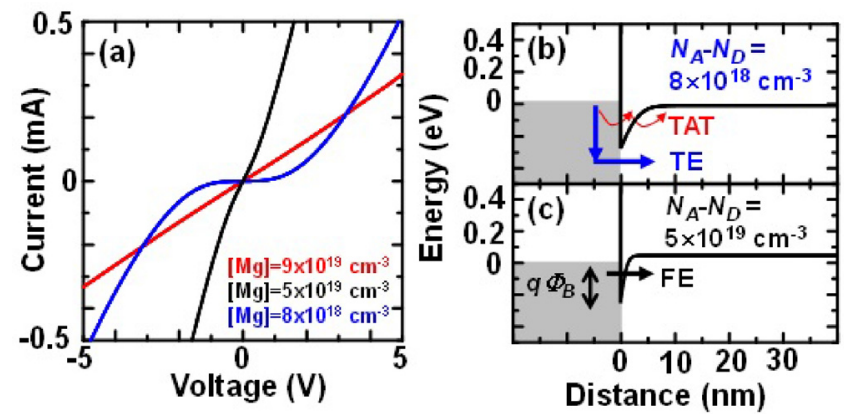

FIG. 1. (a) Current-voltage characteristics of heavily Mg-doped GaN layers using oxidized $\mathrm{Ni} / \mathrm{Au}$ contact. The electrical characteristics of GaN:Mg layers are categorized into three ranges of $[\mathrm{Mg}] \leq 2 \times 10^{19} \mathrm{~cm}^{-3}$ with Schottky behavior, $3 \times 10^{19} \mathrm{~cm}^{-3} \leq[\mathrm{Mg}] \leq 7 \times 10^{19} \mathrm{~cm}^{-3}$ with fairly ohmic behavior, and $9 \times 10^{19} \mathrm{~cm}^{-3} \leq[\mathrm{Mg}]$ with ohmic behavior. (b) Energy band diagram of $p$-type $\mathrm{GaN}\left(N_{A}-N_{D}=8 \times 10^{18} \mathrm{~cm}^{-3}\right)$ at zero voltage bias. In the thermionic emission (TE) model, carriers without enough high thermal energy cannot surmount potential barrier height of $\Phi_{B}$, leading to Schottky characteristics. In the trap-assisted tunneling (TAT) model, thermally activated carriers tunnel through deep-level defects in $p$-type GaN layers. (c) Energy band diagram of $p$-type $\mathrm{GaN}\left(N_{A}-N_{D}=5 \times 10^{19} \mathrm{~cm}^{-3}\right)$ at zero voltage bias. In the field emission (FE) model, a depletion-region width at a metal $/ p^{+}-\mathrm{GaN}$ interface becomes thinner, leading to quantum mechanical tunneling of carriers through the potential barrier.
$P$-type $\mathrm{GaN}$ with $[\mathrm{Mg}]$ above the critical concentration $\left(>7 \times 10^{19} \mathrm{~cm}^{-3}\right)$ displays an ohmic behavior but with a rather high resistance, as indicated by the slope. This very high $[\mathrm{Mg}]$ range is normally used as $p$-type GaN ohmiccontact layers. $P$-type $\mathrm{GaN}$ layers with $[\mathrm{Mg}]$ between $3 \times 10^{19}$ and $7 \times 10^{19} \mathrm{~cm}^{-3}$ show fairly ohmic characteristics with low resistance. In contrast, $\mathrm{GaN}: \mathrm{Mg}$ layers with $[\mathrm{Mg}]$ $\leq 2 \times 10^{19} \mathrm{~cm}^{-3}$ exhibit a Schottky behavior.

Current transport across metal-semiconductor contacts with low impurity concentrations is dominated by thermionic emission (TE). ${ }^{24,25}$ In the TE model (Fig. 1(b)), some of the thermally activated carriers have a larger energy than the top of the potential barrier and conduct from metal into semiconductors, while the other carrier conductance is limited by the potential barrier and the large depletion width at the metal-semiconductor interface, showing a Schottky behavior. On the other hand, the field emission (FE) mechanism is predominant for $p$-type semiconductors with high $N_{A}-N_{D}$. Indeed, the depletion width at the metal-semiconductor interface decreases when increasing $N_{A}-N_{D}$, which results in high carrier-tunneling probability through the potential barrier, as schematically shown in Fig. 1(c). The characteristic energy related to the tunneling probability is defined by $E_{0 O}=q h /(4 \pi)\left(N_{A} / m^{*} \varepsilon_{s}\right)^{1 / 2},{ }^{24}$ where $h$ is Planck's constant, $m^{*}$ the effective hole mass $\left(=1.25 m_{0}\right)$, and $\varepsilon_{s}$ the dielectric constant $\left(=9.8 \varepsilon_{0}\right) .{ }^{26}$ Heavily $\mathrm{Mg}$-doped GaN with $N_{A}>2 \times 10^{19} \mathrm{~cm}^{-3}$ leads to $E_{O O}>k T$ at room temperature, where $k$ is Boltzmann's constant and $T$ the absolute temperature, implying that FE current is dominant. ${ }^{24}$ Notice that this $N_{A}$ value of $2 \times 10^{19} \mathrm{~cm}^{-3}$ corresponds to the transition between Schottky and fairly ohmic characteristics observed on $I-V$ curves of Fig. 1(a).

The resistivity of $0.5-\mu$ m-thick GaN:Mg layers with various $[\mathrm{Mg}]$ is shown in Fig. 2. The resistivity of $p$-type $\mathrm{GaN}$ layers with $[\mathrm{Mg}]$ ranging between $3 \times 10^{19}$ and $7 \times 10^{19} \mathrm{~cm}^{-3}$ decreases with increasing $[\mathrm{Mg}]$, while that of $p$-type $\mathrm{GaN}$ layers for $[\mathrm{Mg}] \geq 9 \times 10^{19} \mathrm{~cm}^{-3}$ gets worse. The lowest layer resistivity is $3 \times 10^{-1} \Omega \mathrm{cm}$ for $[\mathrm{Mg}]=7$ $\times 10^{19} \mathrm{~cm}^{-3}$, which corresponds to the maximum $N_{A}-N_{D}$.

The layer resistivity of a $p$-type semiconductor is given by $\left(q \mu_{p} p\right)^{-1}$ from the drift current under an applied electric field, ${ }^{24}$ where $q$ is the hole charge, $\mu_{p}$ the free hole mobility, and $p$ the hole concentration. Hole mobility and hole concentration are significantly affected by impurity and acceptor

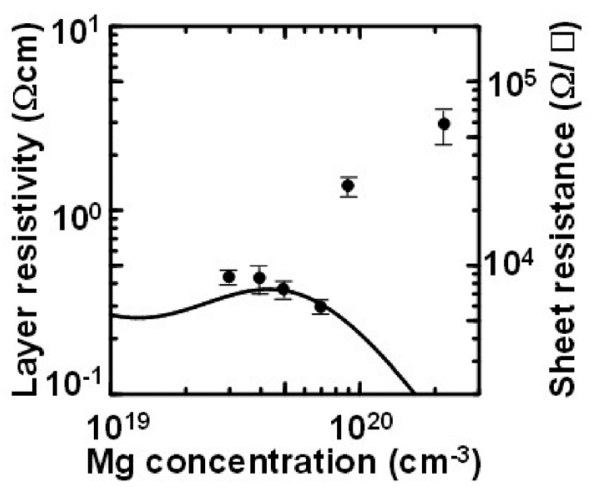

FIG. 2. Relation between layer resistivity and Mg concentration in 500-nmthick GaN:Mg layers. A black line shows layer resistivity estimated from equation of $\left(q \mu_{p} p\right)^{-1}$ using Hall-effect measurements. 
concentrations. According to the empirical model proposed by Caughey-Thomas for silicon, ${ }^{27} \mu_{p}$ at room temperature is given by $\mu_{\text {min }}+\left(\mu_{\text {max }}-\mu_{\text {min }}\right) /\left(1+\left(N_{A}-N_{D} / N_{\text {ref }}\right)^{\beta}\right)$, where $\mu_{\text {min }}, \mu_{\text {max }}$, $N_{\text {ref }}$, and $\beta$ are fitting parameters. The fitting curve of $\mu_{p}$ depending on $N_{A}-N_{D}$ is obtained from Hall-effect measurements for $p$-type $\mathrm{GaN}$ layers with various $[\mathrm{Mg}]$. Meanwhile, the hole concentration in the non-degenerate $p$-type semiconductor is given from the charge-neutrality conditions by $p+N_{D} /\left(N_{A}-N_{D}-p\right)=N_{V} / g \cdot \exp \left(-E_{a} / k T\right)$, where $N_{v}$ is the effective density of states in the valence band and $g$ the acceptor degeneracy. Acceptor ionization energy $E_{a}$ is reduced in proportion to the average distance between ionized acceptors due to binding energy reduction from Coulomb interaction with ionized acceptors and screening of the Coulomb potential by mobile charges. ${ }^{28,29}$ Thus, $E_{a}$ is given by $E_{a}=E_{a 0}+f q^{2}$ $\left(4 \pi / 3 N_{A}{ }^{-}\right)^{1 / 3} /\left(4 \pi \varepsilon_{s}\right)$, where $E_{a 0}$ is the acceptor ionization energy in the absence of ionized acceptor, $\varepsilon_{s}$ the permittivity, and $f$ a geometric factor. Using these equations, we fitted the hole-mobility and hole-concentration dependences as a function of $N_{A}-N_{D}$ with $3 \times 10^{19} \mathrm{~cm}^{-3} \leq[\mathrm{Mg}] \leq 7 \times 10^{19} \mathrm{~cm}^{-3}$ and deduced the layer resistivity. Detailed fitting parameters for these equations were reported elsewhere. ${ }^{19}$ As shown in Fig. 2, the layer resistivity obtained from TLMs qualitatively agrees with the calculated values for $p$-type $\mathrm{GaN}$ layers with $3 \times 10^{19} \mathrm{~cm}^{-3} \leq[\mathrm{Mg}] \leq 7 \times 10^{19} \mathrm{~cm}^{-3}$. On the other hand, $p$-type GaN layers with very high $[\mathrm{Mg}] \geq 9 \times 10^{19} \mathrm{~cm}^{-3}$ have much higher resistivity than the calculated values. We suppose that hole concentration and hole mobility in those layers are strongly reduced due to $\mathrm{Mg}$-acceptor compensation and crystalline-quality degradation.

The relationship between $[\mathrm{Mg}]$ and $\rho_{c}$ is shown in Fig. 3(a). Despite data dispersion, one can see that $\rho_{c}$ first decreases with increasing $[\mathrm{Mg}]$. A minimum value of $2 \times 10^{-5} \Omega \mathrm{cm}^{2}$ is reached for $[\mathrm{Mg}]=5 \times 10^{19} \mathrm{~cm}^{-3}$, which is smaller than the highest $N_{A}-N_{D}\left(=7 \times 10^{19} \mathrm{~cm}^{-3}\right)$ we measured. We suppose that $\mathrm{Mg}$ segregation at surface forms a thin top GaN layer with slightly more $[\mathrm{Mg}]$. The increase of $\rho_{c}$ for $p$-type $\mathrm{GaN}$ with $[\mathrm{Mg}] \geq 9 \times 10^{19} \mathrm{~cm}^{-3}$ is attributed to the decrease of $N_{A}-N_{D}$ due to $\mathrm{Mg}$-acceptor compensation.

When the FE process applies, $\rho_{c}$ is given by $\mathrm{d} V /\left.\mathrm{d} J\right|_{V \rightarrow 0}$. Forward FE-current density has a linear relation with tunneling probability through the potential barrier. Using the Wentzel-Kramers-Brillouin approximation, $\rho_{c}$ behaves as $\rho_{c} \propto \exp \left[\Phi_{B} / E_{0 O}\right]$ for heavily doped semiconductors. ${ }^{24}$
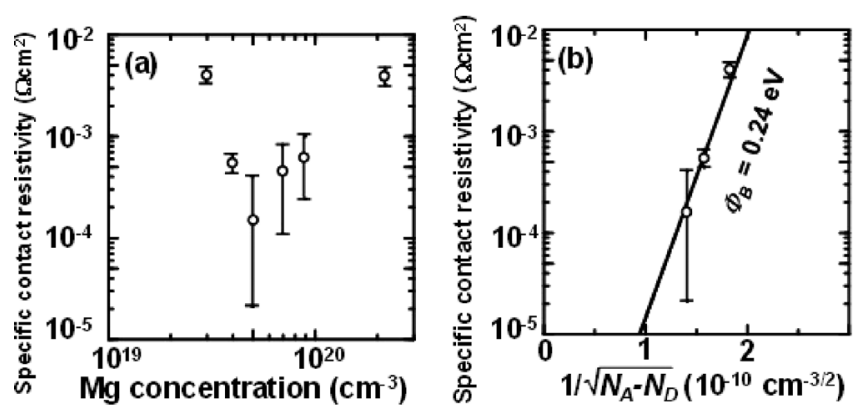

FIG. 3. (a) Dependence of specific contact resistivity on $\mathrm{Mg}$ concentration in 500-nm-thick GaN:Mg layers. (b) Dependence of specific contact resistivity on $\left(N_{A}-N_{D}\right)^{-0.5}$ in 500-nm-thick GaN:Mg layers. Experimental data for $\mathrm{NiO} / p-\mathrm{GaN}$ were fitted in the range of $N_{A}-N_{D}=3-5 \times 10^{19} \mathrm{~cm}^{-3}$ using $\rho_{c} \propto \exp \left[\Phi_{B} / k T\right]$ with $\Phi_{B}=0.24 \mathrm{eV}$.
The experimental data are fitted in the range of $N_{A}-N_{D}$ $=3-5 \times 10^{19} \mathrm{~cm}^{-3}$, as shown in Fig. 3(b). We obtain a linear relation between $\ln \rho_{c}$ and $N_{A}^{-1 / 2}$, indicating that FE current dominates at room temperature in this $[\mathrm{Mg}]$ range. By ignoring image-force lowering, the barrier height at the $\mathrm{NiO} /$ $p^{+}$-GaN interface is extracted to be $0.24 \mathrm{eV}$, in agreement with other reports. ${ }^{12,30}$ We conclude that both low $\Phi_{B}$ $(\sim 0.24 \mathrm{eV})$ and high $N_{A}\left(>2 \times 10^{19} \mathrm{~cm}^{-3}\right)$ ensure ohmic behavior through FE tunneling.

Current-temperature $(I-T)$ measurements were carried out on GaN:Mg layers with $[\mathrm{Mg}]=5 \times 10^{19}$ and $9 \times 10^{19} \mathrm{~cm}^{-3}$ using the circular TLM with a contact pad of $200 \mu \mathrm{m}$ diameter (Fig. 4(a)). The bias voltage was $5 \mathrm{~V}$ for temperatures between 180 and $540 \mathrm{~K}$. In the whole temperature range, higher current is obtained for $\mathrm{GaN}: \mathrm{Mg}$ layers with $[\mathrm{Mg}]=5 \times 10^{19} \mathrm{~cm}^{-3}$. Temperature dependence of layer resistivity and hole concentrations in $p$-type $\mathrm{GaN}$ with $[\mathrm{Mg}]$ of $5 \times 10^{19} \mathrm{~cm}^{-3}$ using the Hall-effect measurement is shown in Fig. 4(b). Measurements were carried out for temperatures between 180 and $310 \mathrm{~K}$, in which $\mathrm{NiO} / p^{+}-\mathrm{GaN}$ contact enables ohmic characteristics. Hole mobility of $p$-type $\mathrm{GaN}$ with $[\mathrm{Mg}]$ of $5 \times 10^{19} \mathrm{~cm}^{-3}$ is almost constant at $1.7 \pm 0.3 \mathrm{~cm}^{2} / \mathrm{Vs}$. The hole concentration significantly increases with increasing temperature due to enhanced acceptor ionization. Thus, the layer resistivity decreases with increasing temperature, contributing to the higher current at high temperature. Here, we should notice that the current in this $[\mathrm{Mg}]$ region depends on not only the layer resistivity but also the type of current transport at a metal-semiconductor interface.

At high temperature, current transport from metal to $p$-type semiconductor includes TE current in addition to FE current or TFE. ${ }^{31}$ In the TFE model, thermally excited carriers enable tunneling through an effective thinner potential barrier in comparison with that in FE current. Current appeared linear on the Arrhenius log-scale plot at high temperature, suggesting a thermally activated mechanism with an $\exp \left(-E_{A} / k T\right)$ functional dependence, where $E_{A}$ is the activation energy of holes above the potential barrier. At high temperature, the hole concentration in $p$-type GaN layers reaches the saturation range $\left(p \sim N_{A}-N_{D}\right){ }^{32}$ By neglecting the change of the hole mobility with increasing temperature, $E_{A}$ is estimated from
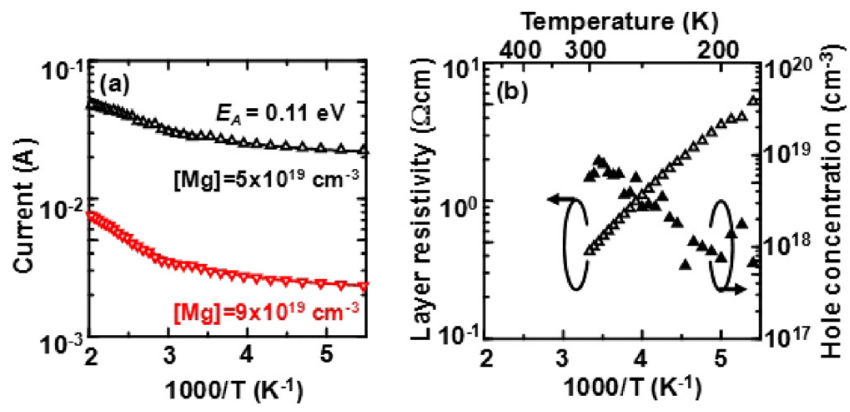

FIG. 4. (a) Temperature-dependent current values of 500-nm-thick GaN:Mg layers with (triangle) $[\mathrm{Mg}]=5 \times 10^{19} \mathrm{~cm}^{-3}$ and (inverted triangle) $[\mathrm{Mg}]=9$ $\times 10^{19} \mathrm{~cm}^{-3}$. Solid lines are fitting values using $\mathrm{FE}$ and $\mathrm{TE}$ models. Experimental data are fitted by equation of $A T+B \exp \left(-E_{A} / k T\right)$, where $A$ and $B$ are different constants. (b) (open triangle) Temperature-dependent layer resistivity of 500-nm-thick GaN:Mg layers with $[\mathrm{Mg}]=4 \times 10^{19}$ and $5 \times 10^{19} \mathrm{~cm}^{-3}$. (closed triangle) Temperature-dependent hole concentrations of that with $[\mathrm{Mg}]=5 \times 10^{19} \mathrm{~cm}^{-3}$. 
fitting the experimental data to be as much as $0.11 \mathrm{eV}$ for $\left[\mathrm{Mg}\right.$ ] between $3 \times 10^{19}$ and $5 \times 10^{19} \mathrm{~cm}^{-3}$, which is almost half of the $\Phi_{B}$ value $(0.24 \mathrm{eV})$ estimated from holeconcentration dependence (Fig. 3(b)). We consider that this small $E_{A}$ in the high temperature regime results from TFE current in addition to the lower layer resistivity.

At low temperature, the dominant current-transport mechanism at the metal $/ p^{+}$-semiconductor interface is $\mathrm{FE}$ tunneling because of insufficient thermal energy for the carriers to go over the potential barrier. Then, the current flow due to hole tunneling from the metal to the $p$-type semiconductor is given by ${ }^{33,34}$

$$
J_{m \rightarrow S}=\frac{A^{*} T}{k} \int_{0}^{q V_{b}} F_{m} p(E)\left(1-F_{s}\right) \mathrm{d} E
$$

where $A^{*}$ is the effective Richardson constant, $q V_{b}$ is the energy difference between the top of potential barrier and the conduction band of undepleted semiconductor regions, $F_{m}$ and $F_{s}$ are the Fermi-Dirac distributions in a metal and semiconductor, respectively, and $E$ is the energy below the top of the potential barrier at the metal-semiconductor interface. Most of the states in the valence band under reverse bias should be unoccupied, yielding $\left(1-F_{s}\right) \sim 1$. The quantum tunneling probability $p(E)$ of holes through the triangular potential barrier is given by $p(E) \approx \exp \left(-4 w \sqrt{2 m^{*} E} / 3 h\right)$, where $w$ is the depletion width at a metal $/ p^{+}$-type $\mathrm{GaN}$ interface and is approximately estimated by $\sqrt{2 \varepsilon_{s B} / q N_{A}} \cdot{ }^{24}$ The estimated value of $\left.p\left(\Phi_{B}\right)\right|_{\mathrm{NA}=5 \times 10^{19}}$ is $2.6 \times 10^{-2}$. We consider that the high $p\left(\Phi_{B}\right)$ in $p^{+}$-type $\mathrm{GaN}$ with $[\mathrm{Mg}]$ between $3 \times 10^{19}$ and $5 \times 10^{19} \mathrm{~cm}^{-3}$ allows FE tunneling, contributing to higher current compared to the situation $[\mathrm{Mg}]$ $=9 \times 10^{19} \mathrm{~cm}^{-3}$ in the whole temperature range.

Let us now discuss the electrical properties for $[\mathrm{Mg}] \geq 9$ $\times 10^{19} \mathrm{~cm}^{-3}$, which exhibit ohmic behavior despite low $N_{A^{-}} N_{D}$ at room temperature. Toward $p$-type GaN with low $N_{A^{-}}$ $N_{D}$, we expect low $p(E)$ through potential barrier at a metal/ $p^{+}$-GaN interface, e.g., $\left.p\left(\Phi_{B}\right)\right|_{\mathrm{NA}=1 \times 10^{18}}=5 \times 10^{-12}$. Thus, FE current cannot flow anymore and a Schottky behavior due to TE current should occur at room temperature. As the other current transport, the ohmic characteristic, or seemingly low potential barrier, can be achieved by tunneling through deeplevel defects in p-type GaN layers and interfacial traps of compounds including accumulated $\mathrm{Mg}$ on the surface, i.e., by trapassisted tunneling (TAT). ${ }^{35,36}$ According to the TAT model, holes conduct from metal through trap states at the $\mathrm{NiO} / p^{+}$GaN interface and in GaN layers (Fig. 1(b)). TAT current requires thermal energy for activation of holes into trap states and/or for hopping conductance through trap states. As shown in Fig. 4(a), total $E_{A}$ at high temperature for $[\mathrm{Mg}] \geq 9 \times 10^{19} \mathrm{~cm}^{-3}$ is estimated to be $0.15 \mathrm{eV}$, which is between $E_{A}$ of $0.11 \mathrm{eV}$ in $[\mathrm{Mg}]$ between $3 \times 10^{19}$ and $5 \times 10^{19} \mathrm{~cm}^{-3}$ and the estimated $\Phi_{B}$ value of $0.24 \mathrm{eV}$. We consider that dominant current in $[\mathrm{Mg}] \geq 9 \times 10^{19} \mathrm{~cm}^{-3}$ includes both TAT and TE, leading to higher $E_{A}$ and $\rho_{c}$ than that in $[\mathrm{Mg}]$ between $3 \times 10^{19}$ and $5 \times 10^{19} \mathrm{~cm}^{-3}$. TAT current through defects has less control. We suggest that achievement of higher $N_{A^{-}} N_{D} \geq 9 \times 10^{19} \mathrm{~cm}^{-3}$ and other approaches, such as the reduction of the potential barrier using suitable metals and narrower band-gap contact layers, would be required for further reduction of $\rho_{c}$.

In conclusion, we systematically investigated the contact resistance in $p$-type GaN. Heavily Mg-doped GaN epilayers with a net acceptor concentration $\left(N_{A}-N_{D}\right)$ in the high $10^{19} \mathrm{~cm}^{-3}$ range were grown using ammonia MBE. The potential-barrier height of the $\mathrm{NiO} / p^{+}-\mathrm{GaN}$ interface was extracted to be $0.24 \mathrm{eV}$. The lowest specific contact resistance $\left(\rho_{c}\right)$ was $2 \times 10^{-5} \Omega \mathrm{cm}^{2}$ for $N_{A^{-}} N_{D}$ of $5 \times 10^{19} \mathrm{~cm}^{-3}$. This low contact resistance is attributed to the hole tunneling through the potential barrier at the metal-GaN interface. Further high $\mathrm{Mg}$ doping reduced $N_{A}-N_{D}$ leading to larger $\rho_{c}$ despite ohmic behavior. This latter behavior is explained by the trap-assisted tunneling process.

This work was supported by the CTI Project 10708.1 PFNM-NM and JSPS KAKENHI Grant No. 15H06070. We would like to acknowledge Dr. Marco Malinvrrni for fruitful discussion.

${ }^{1}$ I. C. Kizilyalli, T. Prunty, and O. Aktas, IEEE Electron Devices Lett. 36, 1073 (2015).

${ }^{2}$ H. Ohta, N. Kaneda, F. Horikiri, Y. Narita, T. Yoshida, T. Mishima, and T. Nakamura, IEEE Electron Devices Lett. 36, 1180 (2015).

${ }^{3}$ N. Kaji, H. Niwa, J. Suda, and T. Kimoto, IEEE Trans. Electron Devices 62, 374 (2015).

${ }^{4}$ T. Kimoto, Jpn. J. Appl. Phys., Part 1 54, 040103 (2015).

${ }^{5}$ V. Adivarahan, A. Lunev, M. A. Khan, J. Yang, G. Simin, M. S. Shur, and R. Gaska, Appl. Phys. Lett. 78, 2781 (2001).

${ }^{6}$ L. Chen, J. Ho, F. Chen, J. Kai, K. Chang, C. Jong, C. Chiu, C. Huang, and K. Shih, Phys. Status Solidi A 176, 773 (1999).

${ }^{7}$ I. Chary, B. Borisov, V. Kuryatkov, Y. Kudryavtsev, R. Asomoza, S. Nikishin, and M. Holtz, MRS Proceedings 1108, A09-30 (2008).

${ }^{8}$ J. Kwak, O. Nam, and Y. Park, Appl. Phys. Lett. 80, 3554 (2002).

${ }^{9}$ M. Meneghini, L. Rigutti, L. R. Trevisanello, A. Cavallini, G. Meneghesso, and E. Zanoni, J. Appl. Phys. 103, 063703 (2008).

${ }^{10}$ J. K. Kim, J.-L. Lee, J. W. Lee, H. E. Shin, Y. J. Park, and T. Kim, Appl. Phys. Lett. 73, 2953 (1998).

${ }^{11}$ X. A. Cao, S. J. Pearton, G. Dang, A. P. Zhang, F. Ren, and J. M. Van Hove, Appl. Phys. Lett. 75, 4130 (1999).

${ }^{12}$ J. O. Song, J.-S. Ha, and T.-Y. Seong, IEEE Trans. Elect. Devices 57, 42 (2010).

${ }^{13}$ Y.-L. Li, E. F. Schubert, J. W. Graff, A. Oshinsky, and W. F. Schaff, Appl. Phys. Lett. 76, 2728 (2000).

${ }^{14}$ P. Vennéguès, M. Benaissa, S. Dalmasso, M. Leroux, E. Feltin, P. D. Mierry, B. Beaumont, B. Damilano, N. Grandjean, and P. Gibart, Mater. Sci. Eng. B 93, 224 (2002).

${ }^{15}$ G. Koblmuller, S. F. Garrido, E. Calleja, and J. S. Speck, Appl. Phys. Lett. 91, 161904 (2007).

${ }^{16}$ H. Okumura, B. M. McSkimming, T. Huault, C. Chaix, and J. S. Speck, Appl. Phys. Lett. 104, 012111 (2014).

${ }^{17}$ E. Trybus, W. A. Doolittle, M. Moseley, W. Henderson, D. Billingsley, G. Namkoong, and D. C. Look, Phys. Status Solidi C 6, S788 (2009).

${ }^{18}$ M. Malinverni, J. M. Lamy, D. Martin, E. Feltin, J. Dorsaz, A. Castiglia, M. Rossetti, M. Duelk, C. Velez, and N. Grandjean, App. Phys. Lett. 105, 241103 (2014).

${ }^{19}$ H. Okumura, D. Martin, M. Malinverni, and N. Grandjean, Appl. Phys. Lett. 108, 072102 (2016).

${ }^{20}$ J.-L. Lee, J. K. Kim, J. W. Lee, Y. J. Park, and T. Kim, Solid State Electron. 43, 435 (1999).

${ }^{21}$ J.-K. Ho, C.-S. Jong, C. C. Chiu, C.-N. Huang, K.-K. Shih, L.-C. Chen, F.-R. Chen, and J.-J. Kai, J. Appl. Phys. 86, 4491 (1999).

${ }^{22}$ A. Castiglia, J.-F. Carlin, and N. Grandjean, Appl. Phys. Lett. 98, 213505 (2011).

${ }^{23}$ G. Miceli and A. Pasquarello, Phys. Rev. B 93, 165207 (2016).

${ }^{24}$ S. M. Sze, Physics of Semiconductor Devices, 3rd ed. (Wiley, New York, 1981). 
${ }^{25}$ G. Greco, P. Prystawko, M. Leszczynski, R. L. Nigro, V. Raineri, and F. Roccaforte, J. Appl. Phys. 110, 123703 (2011).

${ }^{26}$ B. Santic, Semicond. Sci. Technol. 18, 219 (2003).

${ }^{27}$ D. M. Caughey and R. E. Thomas, Proc. IEEE 55, 2192 (1967).

${ }^{28}$ G. L. Pearson and J. Bardeen, Phys. Rev. 75, 865 (1949).

${ }^{29}$ P. P. Debye and E. M. Conwell, Phys. Rev. 93, 693 (1954).

${ }^{30}$ H. Ishikawa, S. Kobayashi, Y. Koide, S. Yamasaki, S. Nagai, J. Umezaki, M. Koike, and M. Murakami, J. Appl. Phys. 81, 1315 (1997).
${ }^{31}$ T. Hashizume, J. Kotani, and H. Hasegawa, Appl. Phys. Lett. 84, 4884 (2004).

${ }^{32} \mathrm{H}$. Morkoc, Nitride Semiconductor Devices (Wiley-VCH, 2013).

${ }^{33}$ H. Yamamoto, Z.-Q. Fang, and D. C. Look, Appl. Phys. Lett. 57, 1537 (1990).

${ }^{34}$ E. J. Miller, E. T. Yu, P. Waltereit, and J. S. Speck, Appl. Phys. Lett. 84, 535 (2004).

${ }^{35}$ D. M. Sathaiya and S. Kamalkar, J. Appl. Phys. 99, 093701 (2006).

${ }^{36}$ Y. Park and H. Kim, Appl. Phys. Exp. 4, 085701 (2011). 